\title{
A Two-Layered Model for Dynamic Supply Chain Management Considering Transportation Constraint ${ }^{*}$
}

\author{
Yoshitaka TANIMIZU ${ }^{* *}$, Kana HARADA ${ }^{* * *}$, Chisato OZAWA ${ }^{* *}$, \\ Koji IWAMURA $^{* *}$ and Nobuhiro SUGIMURA ${ }^{* *}$ \\ **Graduate School of Engineering, Osaka Prefecture University, \\ ${ }^{* * *}$ School of Engineering, Osaka Prefecture University, \\ 1-1 Gakuen-cho Naka-ku Sakai, Osaka 599-8531, Japan \\ E-mail: tanimizu@me.osakafu-u.ac.jp
}

\begin{abstract}
This research proposes a two-layered model for dynamic supply chain management considering transportation constraint. The model provides a method for suppliers to estimate suitable prices and delivery times of products based on not only production schedules but also transportation plans in consideration of constraints about shipping times and loading capacities for transportation. A prototype of dynamic supply chain simulation system was developed and some computational experiments were carried out in order to verify the effectiveness of the model. The prototype system is available to determine suitable shipping times and loading capacities of transportation vehicles.
\end{abstract}

Key words: Supply Chain Management, Scheduling, Transportation, Genetic Algorithm, Negotiation

\section{Introduction}

Supply chain management has been investigated, in recent years, for planning and configuring of information flow and material flow among different organizations, such as raw material suppliers, parts suppliers and assembly manufacturers ${ }^{(1)}$. Recent information technologies provide us with global and fast communication among the different organizations. Therefore, each organization requires a strategy for dynamically changing the configuration and finding suitable business partners for every order in order to enter into proper contracts with the partners and make profits to survive in the dynamic supply chain $^{(2-6)}$.

Previous researches ${ }^{(7-8)}$ proposed a two-layered supply chain model consisting of a set of clients and suppliers. The proposed model provided a systematic method to estimate suitable prices and delivery times of products through the iteration of both the modification processes of production schedules of the suppliers by using a genetic algorithm $(\mathrm{GA})^{(9-11)}$ and the negotiation processes among the clients and the suppliers.

This research extends the model for dynamic supply chain management considering transportation constraint. The extended model provides a method for the suppliers to estimate suitable prices and delivery times of products based on not only production schedules but also transportation plans. A prototype of dynamic supply chain simulation system was developed and some computational experiments were carried out in order to verify the effectiveness of the model. The prototype system is available to determine suitable shipping times and loading capacities of transportation vehicles.

Feb., 2010 (No. 10-0071)

Copyright () 2010 by JSME 


\section{Dynamic Supply Chain Management}

\subsection{Framework}

The organizations in the dynamic supply chains are generally classified into two kinds of organizations from the viewpoint of pushing/pulling parts or products, as shown in Fig. 1. One organization has a make-to-stock (MTS) production system which maintains stock of assembled parts or products. The organization makes orders and assembles parts or products for keeping the stock as small as possible without creating shortages. The other organization has a make-to-order (MTO) or build-to-order (BTO) production system which has no stock of assembled parts or products. The organization assembles parts or products only after they are ordered.

The target of this research is a group of organizations having MTO or BTO production systems in the dynamic supply chain. Suitable prices and delivery times of ordered products are determined through a systematic method developed in the research. Recent researches in this type of dynamic supply chains involve using multi-agent systems and combinatorial auctions. The agent in the supply chain negotiates with its neighbour agents in order to determine prices and delivery times.

\subsection{Two-Layered Models for Dynamic Supply Chain Management}

The previous researches ${ }^{(7-8)}$ proposed a two-layered supply chain model, which consists of two components of the clients and the suppliers, as shown in Fig. 2. An auction-based method was proposed, in the papers, to make decisions and to negotiate among the clients and the suppliers. Suitable prices and delivery times of products are determined through the iteration of both the modification processes of production schedules of the suppliers by using a GA and the negotiation processes among the clients and the suppliers.

Let us consider negotiation processes between a client and a set of suppliers for a newly ordered product. Figure 3 summarizes the negotiation processes for determining the price and the delivery time of the newly ordered product between the client and the suppliers. The followings are steps of the negotiation processes between the client and the suppliers.

1. The client generates the new order of the product $N(C, p, n)$ and sends it to all the suppliers, when the client needs the product $N(C, p, n)$.

2. The suppliers have production schedules including the pre-ordered products $G(S, q, g)$ which have already been contracted. The suppliers modify the existing production schedules including the newly ordered product $N(C, p, n)$, and generate

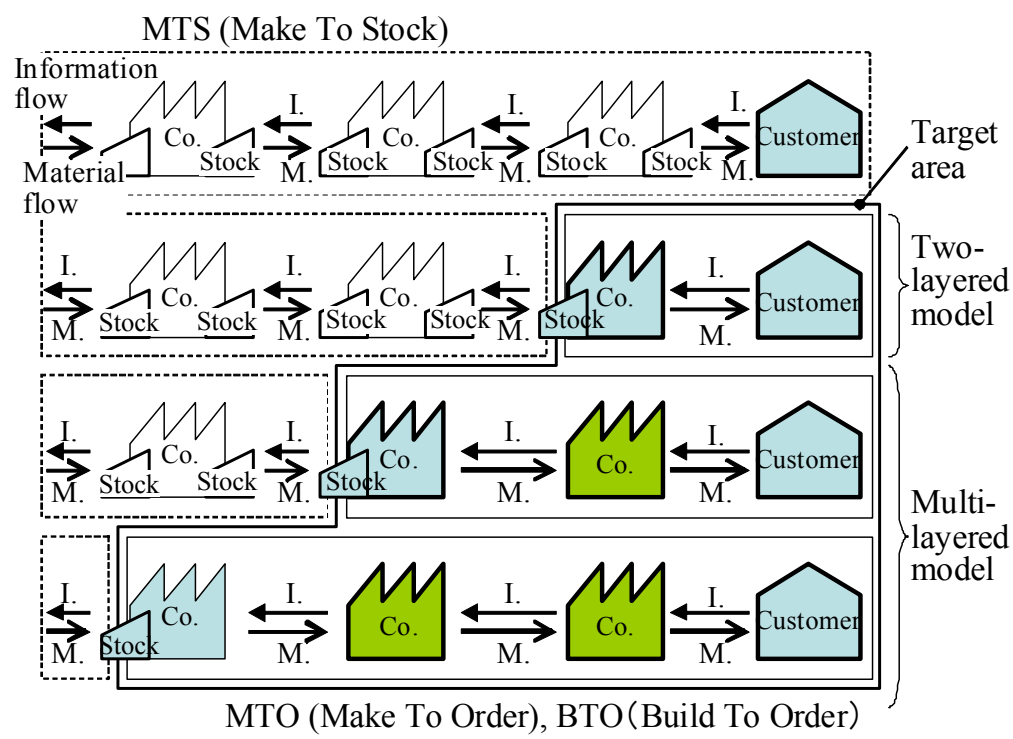

Fig. 1 Types of supply chains 
the offers sent to the client. The offers include the information about the bid price and the possible delivery time of the newly ordered product $N(C, p, n)$.

3. The client evaluates all the offers sent from the suppliers. The client accepts the most suitable offer, when the offer satisfies the requirements on both the price and the delivery time of the newly ordered product. However, if no offers match with the order, the client relaxes the requirements of the order and sends it to the suppliers.

The negotiation processes are repeated until an offer satisfies the order and one of the suppliers enters into a contract with the client.

\section{Extension of Model Considering Transportation Constraint}

\subsection{Transportation Constraint}

This research extends the two-layered supply chain model to develop a new model of dynamic supply chains considering transportation constraint. The extended model provides a method for the suppliers to estimate suitable prices and delivery times of products based on not only production schedules but also transportation plans.

The following conditions are assumed as transportation constraint in the model.

- A product is transported from a supplier to a client by a transportation vehicle.

- Transportation vehicles do not travel to two or more clients in one delivery.

- Each transportation vehicle leaves a supplier at constant time interval and transports products in a given time.

- The capacity for loading products is limited on a transportation vehicle. The

Supplier: $U_{S}$

Client: $U_{C}$

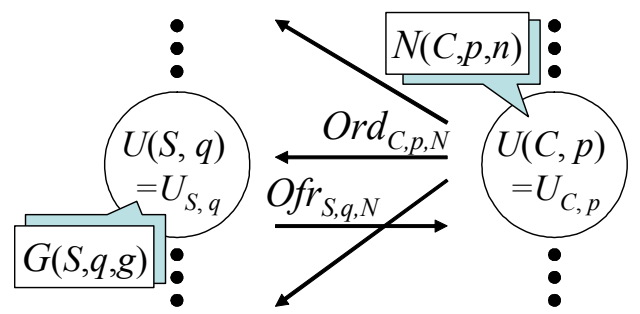

Fig. 2 Two-layered supply chain model

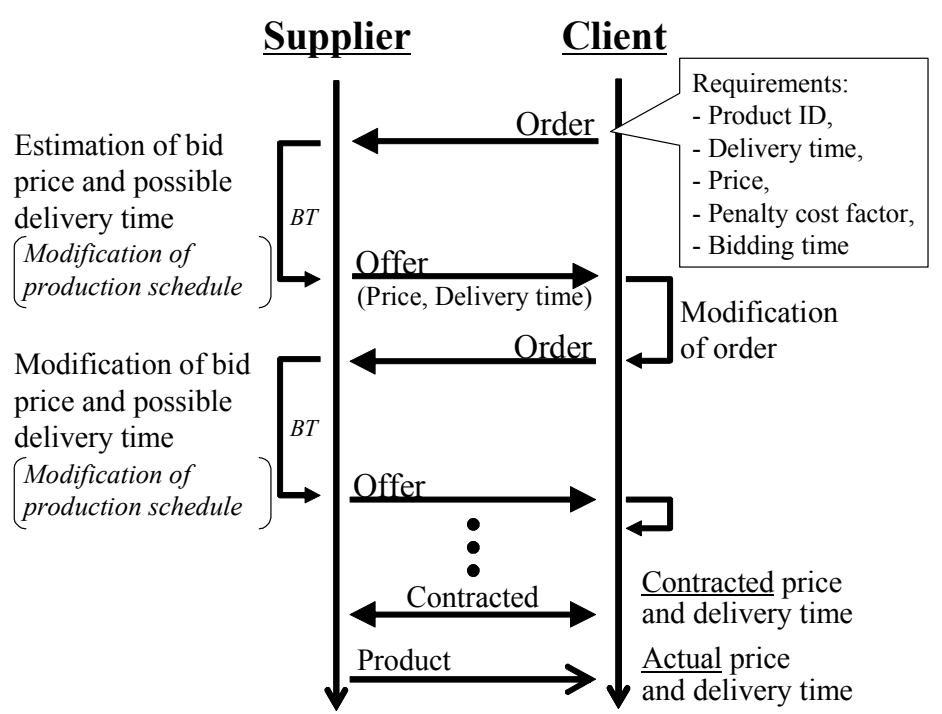

Fig. 3 Negotiation process between clients and suppliers 
amount of products loaded on a vehicle is less than or equal to the limit of loading capacity.

The following terms are defined in this research to describe the model of two-layered dynamic supply chains considering transportation constraint.

- Transportation time $\left(T T_{q, p}\right)$ : Time it takes to transport products from the supplier $U_{S, q}$ to the client $U_{C, p}$. A transportation path is specified and the transportation time is determined by a combination of the client and the supplier.

- Shipping time $\left(H T_{q, p}^{v}\right)$ : Time that the transportation vehicle leaves the supplier $U_{S, q}$ for the client $U_{C, p}$ at the $v$-th shipping of products.

- Interval of time between shipping $\left(I T H_{q, p}\right)$ : Interval of time between the $v$-th shipping time $H T_{q, p}^{v}$ and the $(v+1)$-th shipping time $H T_{q, p}^{v+1}$. This research assumes that the transportation vehicles leave the supplier at constant time interval. It is represented by the following condition. An upper limit of the shipping frequency can be calculated from the time interval.

$$
I T H_{q, p}=H T_{q, p}^{v+1}-H T_{q, p}^{v}
$$

- Maximum loading capacity $\left(M L D_{q, p}\right)$ : Upper limit of amount of products that can be loaded to a transportation vehicle for shipping from the supplier $U_{S, q}$ to the client $U_{C, p}$.

- Loading amount $\left(L D_{q, p}^{v}\right)$ : Amount of products that are loaded on the transportation vehicle at the $v$-th shipping from the supplier $U_{S, q}$ to the client $U_{C, p}$. The transportation vehicles are not able to load products over the maximum loading capacity $M L D_{q, p}$. In the case where the loading amount $L D_{q, p}^{v}$ is 0 , the transportation vehicle does not leave the supplier. Therefore, the following condition should be considered.

$0<L D_{q, p}^{v} \leq M L D_{q, p}$

- $\quad$ Room for loading $\left(R L D_{q, p}^{v}\right)$ : Amount of room on the transportation vehicle which leaves the supplier $U_{S, q}$ to the client $U_{C, p}$ at the $v$-th shipping. The room for loading is described by the following equation.

$$
R L D_{q, p}^{v}=M L D_{q, p}-L D_{q, p}^{v}
$$

- $\quad$ Shipping cost per vehicle $\left(H C V_{q, p}\right)$ : Cost of a transportation from the supplier $U_{S, q}$ to the client $U_{C, p}$ by the transportation vehicle. It is not dependent on the loading amount, but the transportation time and the maximum loading capacity of the transportation vehicle.

- $\quad$ Shipping cost per product $\left(H C_{q, p}\right)$ : Cost of a transportation per product delivered from the supplier $U_{S, q}$ to the client $U_{C, p}$. It is expressed in the following equation.

$$
H C_{q, p}=\frac{H C V_{q, p}}{M L D_{q, p}}
$$

The transportation plans generally include constraints about shipping times and loading capacities for transportation. The ordered products are transported by the transportation vehicles at constant time intervals, after the suppliers finish manufacturing the products. The amount of loading products on the transportation vehicles must not be over specified maximum loading capacities. 


\subsection{Estimation of Possible Delivery Time}

This research proposes a method for estimating the delivery times of products by allocating the products to proper transportation vehicles within the maximum loading capacities in the order of the completion times of the products. Two cases are considered for the estimation of the possible delivery time, in the case where the following equation describes the completion time of the product in relation to the shipping times of vehicles.

$$
H T_{q, p}^{v-1}<\max \left\{f t_{S, q, N}\right\} \leq H T_{q, p}^{v}
$$

(1) $L D_{q, p}^{v} \leq M L D_{q, p}$

When the loading amount of the transportation vehicle at the $v$-th shipping is less than the maximum loading capacity, the product can be loaded on the transportation vehicle. Then, the possible delivery time of the product is given by the following equation.

$$
D T_{S, q, N}^{O f r}=H T_{q, p}^{v}+T T_{q, p}
$$

(2) $L D_{q, p}^{v}>M L D_{q, p}$

In the case where the loading amount of the transportation vehicle at the $v$-th shipping has already filled to the maximum loading capacity, the product should be assigned to another transportation vehicle which has some room for loading at the $(v+1)$-th or later shipping. Then, the possible delivery time of the product is given by the following equation.

$$
\begin{array}{ll}
D T_{S, q, N}^{O f r}=H T_{q, p}^{v+i}+T T_{q, p} & (i=1,2 \ldots) \\
L D_{q, p}^{v+i} \leq M L D_{q, p} & (i=1,2 \ldots)
\end{array}
$$

Figure 4 shows an example of the estimation processes of possible delivery times. The upper bar chart in Fig. 4 is a gantt chart that represents a production schedule of a supplier, and the lower bar chart in Fig. 4 shows a transportation plan assigning the products to each

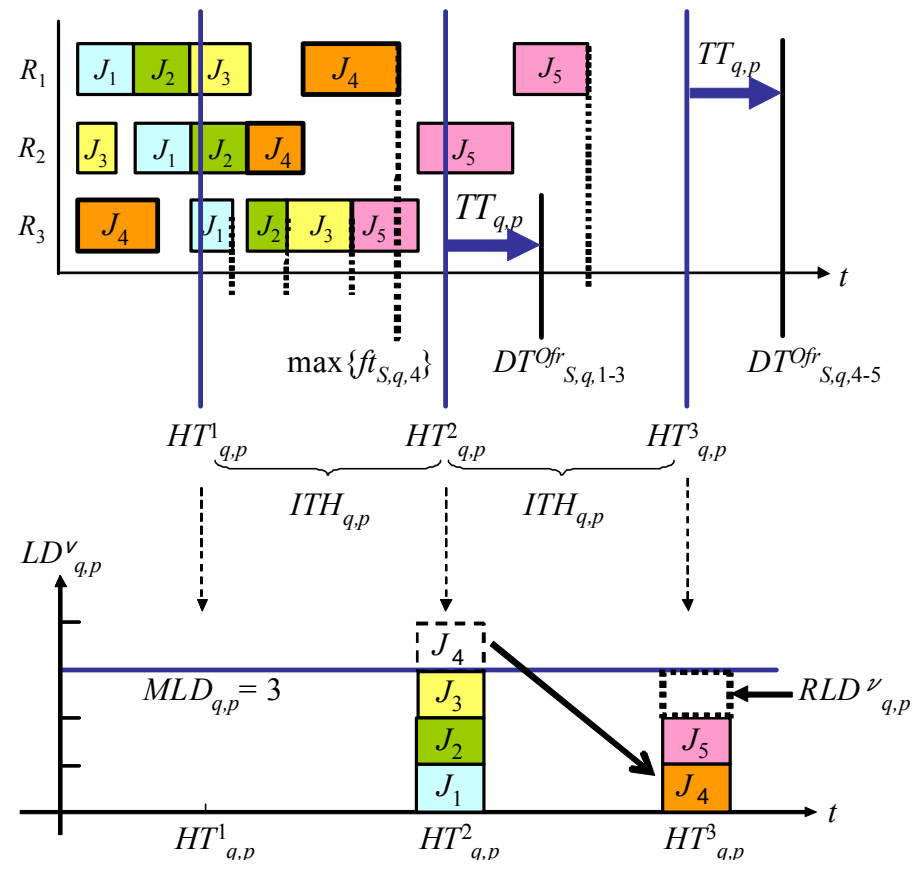

Fig. 4 Estimation processes of delivery times 
transportation vehicle. Let the maximum loading capacity $M L D_{q, p}$ is 3 for the transportation vehicles here. The shipping times of the products $J_{1}, J_{2}$, and $J_{3}$ are determined as $H T_{q, p}^{2}$ because of their finishing times calculated from the production schedule. Therefore, their possible delivery times $D T_{S, q, i}^{O f r}(i=1 \sim 3)$ is estimated as $\left(H T_{q, p}^{2}+T T_{q, p}\right)$. The shipping time of the product $J_{4}$ is normally determined as $H T_{q, p}^{2}$ because of its finishing time. However the transportation vehicle for the second shipping has already filled to the maximum loading capacity with the products $J_{1}, J_{2}$, and $J_{3}$, and the transportation vehicles are not able to load the products over the maximum loading capacity. Therefore, the product $J_{4}$ is transported by the next transportation vehicle, thus, the shipping time of the product $J_{4}$ is determined as $H T_{q, p}^{3}$ and its possible delivery time is estimated as $\left(H T_{q, p}^{3}+T T_{q, p}\right)$.

\subsection{Estimation of Bid Price}

The bid price of the product is estimated by adding the shipping cost per product to the bid prices defined in the previous researches ${ }^{(7-8)}$. Therefore, the bid price of the newly ordered product $N(C, p, n)$ is expressed by the following equation.

$$
P C_{S, q, N}^{O f r}=T C_{S, q, N}+R W_{S, q, N}+H C_{q, p}-P N_{S, q, N}+\sum_{i=1}^{g} \Delta P N_{S, q, G(S, q, i)}^{R v(N, r)}
$$

where,

$T C_{S, q, N} \quad$ Total manufacturing cost including material cost and production cost for the product $N$.

$R W_{S, q, N} \quad$ Reward required for the product $N$.

$H C_{q, p} \quad$ Shipping cost per product.

$P N_{S, q, N} \quad$ Penalty cost of the product $N$ due to delay of offered delivery time to ordered delivery time.

$\Delta P N_{S, q, G}^{R v(N, r)}$ Penalty cost due to delays of delivery times of pre-ordered products $G_{S, q, i}$ $(i=1,2, \ldots, g)$, if the addition of the product $N$ causes the delays.

Equation (9) means that the suppliers add the penalty costs of the pre-ordered products $G_{S, q, i}(i=1,2, \ldots, g)$ to the price of the product $N$, if their delivery times are delayed due to the insertion of the product $N$ in the production schedule. This equation also means that the suppliers discount the price of the product $N$ by the penalty $\operatorname{cost} P N_{S, q, N}$, if the delivery time of the product $N$ does not satisfy the required delivery time given by the client.

\subsection{Estimation of Actual Profit}

The client sends the order to all the suppliers, and receives the offers from the suppliers by the time limit for the offers. The client accepts one of the offers from the suppliers, if both the bit price and the possible delivery time of the offer satisfy the following equations.

$$
\begin{gathered}
D T_{S, q, N}^{O f r} \leq D T_{C, p, N}^{O r d} \\
P C_{S, q, N}^{O f r} \leq P C_{C, p, N}^{O r d}
\end{gathered}
$$

The contracted price and delivery time of the product are obtained by the following equations, when the supplier enters into a contract with the client.

$$
\begin{aligned}
& D T_{S, q, N}^{C n t}=D T_{S, q, N}^{O f r} \\
& P C_{S, q, N}^{C n t}=P C_{S, q, N}^{O f r}
\end{aligned}
$$


The actual price and delivery time are different from the contracted price and delivery time, since the suppliers continuously modify the production schedules in order to enter into a lot of contracts for new orders. The contracted price and delivery time mean the price and the delivery time determined through the negotiation processes with the client. On the other hand, the actual price and delivery time mean the price and the delivery time when the ordered product is delivered to the client. The actual price of the product is expressed by the following equation.

$$
P C_{S, q, N}^{A c t}=P C_{S, q, N}^{C n t}-k_{C, p, N} \times \max \left\{D T_{S, q, N}^{A c t}-D T_{S, q, N}^{C n t}, 0\right\}
$$

The suppliers can estimate the profits, when the suppliers enter into contracts with the clients. However, the actual profit for the ordered product is different from the required reward $R W_{S, q, N}$ used in Eq. (9). The total amount of actual profits $T P F_{S, q}$ is estimated by the following equation.

$$
\begin{aligned}
& T P F_{S, q}=\sum_{h=1}^{p}\left\{\sum_{j=1}^{n}\left(P C_{S, q, N(C, h, j)}^{A c t}-T C_{S, q, N(C, h, j)}\right)-H C V_{q, h} \times v_{q, h}\right\} \\
& =\sum_{h=1}^{p} \sum_{j=1}^{n}\left(P C_{S, q, N(C, h, j)}^{A c t}-T C_{S, q, N(C, h, j)}\right)-\sum_{h=1}^{p} \sum_{f=1}^{v}\left(L D_{q, h}^{f}+R L D_{q, h}^{f}\right) \times H C_{q, h} \\
& =\sum_{h=1}^{p} \sum_{j=1}^{n}\left(R W_{S, q, N(C, h, j)}-P N_{S, q, N(C, h, j)}+\sum_{i=1}^{g} \Delta P N_{S, q, G(S, q, i)}^{R v(N(C, h, j), r)}\right) \\
& \quad-\sum_{h=1}^{p} \sum_{j=1}^{n}\left(k_{C, h, N(C, h, j)} \times \max \left\{D T_{S, q, N(C, h, j)}^{A c t}-D T_{S, q, N(C, h, j)}^{C n t}, 0\right\}\right) \\
& \quad-\sum_{h=1}^{p} \sum_{f=1}^{v}\left(R L D_{q, h}^{f} \times H C_{q, h}\right)
\end{aligned}
$$

Equation (15) represents how the suppliers obtain profits. First of all, the suppliers should enter into contracts to obtain rewards for products. Secondly, the suppliers should reduce the penalty costs that are paid to clients due to the delays of delivery times of products. Thirdly, the suppliers should eliminate wasteful shipping cost by reducing the room for loading on the transportation vehicles, since the suppliers should pay the shipping cost for the room for loading in the case where the loading amounts of transportation vehicles are less than the maximum loading capacities.

The suppliers need suitable transportation constraints, in order not to decrease in profits. The suppliers have to pay a lot of cost for rooms for loading, if the transportation vehicles have much larger loading capacities than the amount of products to be delivered. On the other hand, the suppliers may lose chances to enter into the contracts, if the loading capacities of transportation vehicles are not enough to deliver the products within the required delivery times.

\section{Computational Experiments}

\subsection{Simulation System}

This research extended an existing prototype of simulation system for dynamic supply chain management considering transportation constraint and applied it to computational experiments in order to verify the effectiveness of the proposed model. The existing prototype system had been developed using networked personal computers. Clients and 
suppliers had been implemented as the agents on different computers. The new prototype system provided supplier agents with functions for estimating the bit prices and the possible delivery times of products based on not only production schedules but also transportation plans. Then, the prototype system is available to determine suitable shipping times and loading capacities of transportation vehicles. Three kinds of experiments were carried out in this research.

\subsection{Experiments for Variations of Shipping Times}

The first experiments were carried out on a basic problem which consists of one supplier agent and one client agent. The experiments were repeated by changing the interval of time between shipping, in order to determine suitable shipping times without considering the limits of loading capacities. The experimental conditions are summarized as follows.

- Initial production schedule of supplier ${ }^{(12)}$

- Job-shop type production schedule

- Number of resources: 10

- $\quad$ Number of pre-ordered products: 10

- Transportation time: $20[\mathrm{~min}]$

- Interval of time between shipping: 30, 60 or 90 [min]

- Maximum loading capacity: unlimited

The client agent continuously generated new orders for about 8 hours, and the supplier agent generated offers and negotiated with the client agent during the experiments. Figure 5 shows the loading amounts of transportation vehicles leaving at each shipping time. The experimental results are summarized in Table 1. As the interval of time between shipping became shorter, however, the number of shipping increased and the average of loading amount per vehicle decreased as shown in Table 1 . In the case where the interval of time between shipping was 30 [min], the variance of loading amount was smallest in the three experimental results.

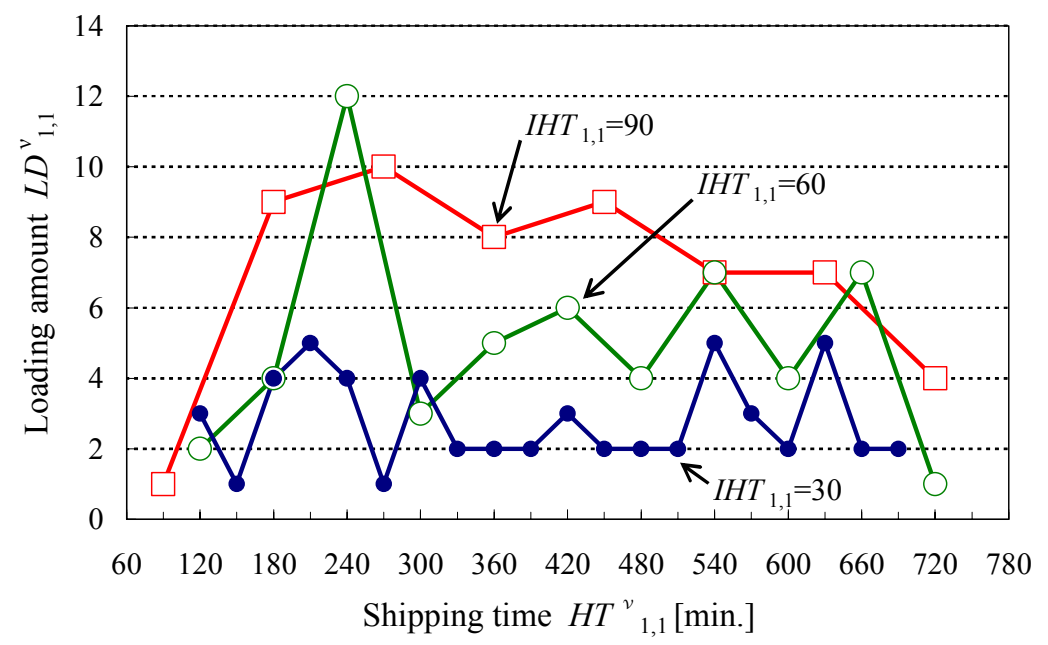

Fig. 5 Loading amount at each shipping time

Table 1 Experimental results for shipping times

\begin{tabular}{|l|l|l|l|}
\hline Interval of time between shipping [min] & 30 & 60 & 90 \\
\hline \hline Total number of contracts & 56 & 55 & 55 \\
\hline Number of shipping & 20 & 11 & 8 \\
\hline Average of loading amount & 2.8 & 5.0 & 6.9 \\
\hline Variance of loading amount & 1.6 & 8.2 & 7.9 \\
\hline
\end{tabular}




\subsection{Experiments for Variations of Loading Capacities}

The second experiments were carried out on the same initial condition with the first experiment. However, the interval of time between shipping was set to 30 [min], in consideration of the variance of loading amount. The upper limit of the maximum loading capacity was set to 5 . The experiments were repeated by changing the value of the maximum loading capacity. The most suitable value of the maximum loading capacity is estimated through the experiments. The experimental results are summarized in Fig. 6 and Table 2.

The supplier was able to enter into about 55 contracts with the client in the experiments, except when the maximum loading capacity of a transportation vehicle was set to 2 . This maximum loading capacity was too small to deliver the products by the required delivery times. Therefore, the supplier lost a lot of chances to enter into contracts. On the other hand, the experimental results show that the excess loading capacity causes the increase of the room for loading and the decrease of the profit. Therefore, it is recognized that the maximum loading capacity should be set to 3 from the viewpoint of the profit of the supplier.

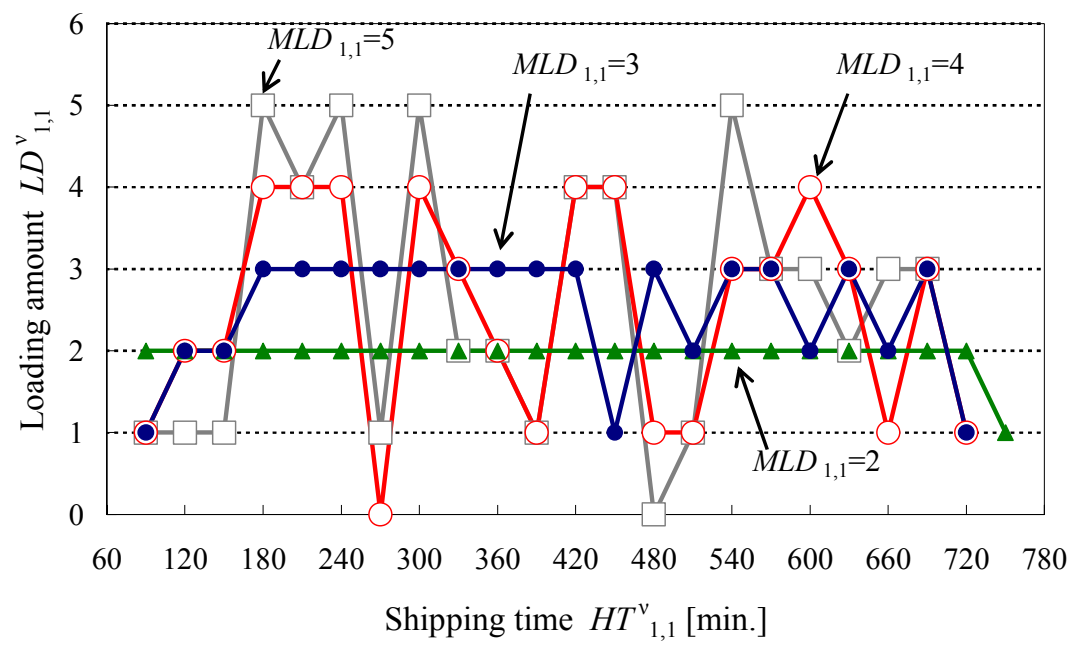

Fig. 6 Loading amount on different loading capacities

Table 2 Experimental results for loading capacities

\begin{tabular}{|l|l|l|l|l|}
\hline Maximum loading capacity & 2 & 3 & 4 & 5 \\
\hline \hline Total number of contracts & 45 & 55 & 55 & 56 \\
\hline Number of shipping & 23 & 22 & 21 & 20 \\
\hline Average of loading amount & 2.0 & 2.5 & 2.5 & 2.7 \\
\hline Summation of room for loading & 1 & 11 & 29 & 44 \\
\hline
\end{tabular}

\subsection{Experiments for Multiple Clients}

The third experiments were carried out using one supplier $U_{S, 1}$ and three clients $U_{C, 1}$, $U_{C, 2}$ and $U_{C, 3}$, as shown in Fig. 7 , in order to evaluate the effectiveness of the proposed model for two or more clients. Three transportation vehicles individually transport the products from the supplier to the three clients in the simulation system. Transportation times $T T_{1,1}, T T_{1,2}$ and $T T_{1,3}$ were set to 15,110 and 150 [min], respectively. Suitable shipping times and loading capacities of the transportation vehicles were determined based on the procedures described in section 4.2 and 4.3.

Firstly, some experiments were carried out by changing the intervals of times between shipping $I H T_{1,1}, I H T_{1,2}$ and $I H T_{1,3}$ for the clients without limitations of the loading 
capacities on the transportation vehicles. Then, the suitable intervals of times were determined as 30, 60 and 90 [min] for $I H T_{1,1}, I H T_{1,2}$ and $I H T_{1,3}$, respectively, from the viewpoint of the minimization of variance of loading amount. Secondly, under the condition that the intervals of times between shipping were set to the determined values, some experiments were carried out by changing the maximum loading capacities on the transportation vehicles. Figure 8 summarizes the experimental results. The horizontal axis shows the variations of the combination of maximum loading capacities. Light-colored bars show the total number of contracts, which the supplier entered into, and darkly-colored bars show the summations of rooms for loading. As the results of the experiments, in the case where $M L D_{1,1}, M L D_{1,2}$ and $M L D_{1,3}$ are set to 2, 2 and 2, respectively, the supplier can enter into much contracts in all the experiments and get less rooms for loading.

The supplier may lose chances to enter into contracts in the case where the intervals of time between shipping are too long and the maximum loading capacities are too small to deliver the products without waiting for another transportation vehicle. On the other hand, the short time intervals of shipping and the large maximum loading capacities cause the decreases in profit of the supplier by paying wasteful costs for transportation. The supplier must pay all the shipping costs per vehicles, even if the transportation vehicles deliver fewer products than the maximum loading capacities.

\section{Conclusion}

This research proposed a two-layered model for dynamic supply chain management considering transportation constraint. The results are summarized as follows.

- The model provides a method for the suppliers to estimate suitable prices and delivery times of products based on not only production schedules but also transportation plans. The transportation plans include constraints about shipping times and loading capacities for transportation.

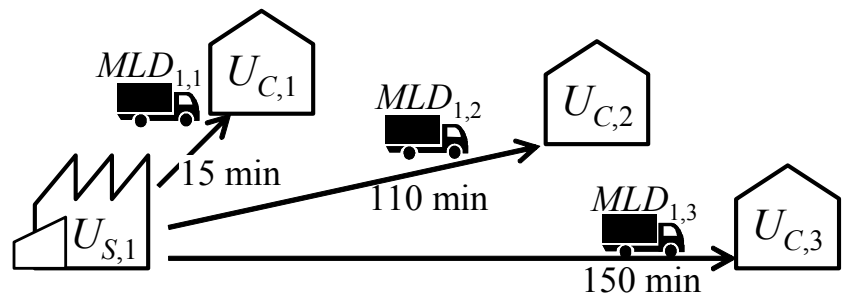

Fig. 7 Simulation model

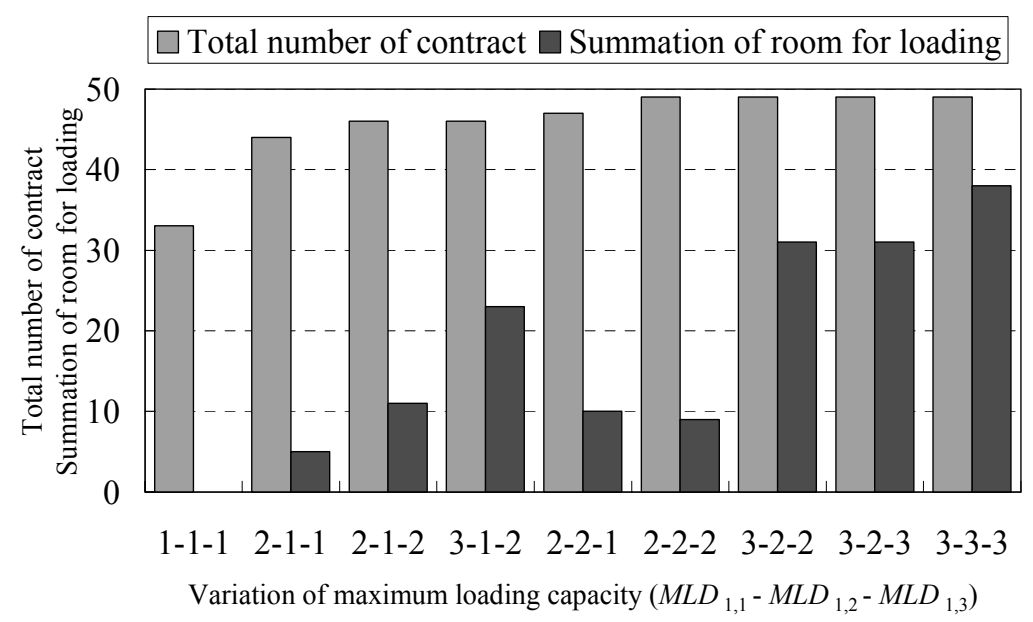

Fig. 8 Experimental results for variations of MLD 
- A prototype of dynamic supply chain simulation system was developed and some computational experiments were carried out in order to verify the effectiveness of the model. The experimental results showed that the prototype system obtained a set of suitable shipping times and loading capacities.

\section{References}

(1) Fujino, N, Essence of Supply Chain and Financial Impact (in Japanese), Harvard Business, Diamond, Vol. 23, No. 6 (1998), pp. 10-21.

(2) Kaihara, T. et al., Virtual Enterprise Negotiation Strategy in Agile Manufacturing Environment, Transactions of the Japan Society of Mechanical Engineers, Series C, Vol. 67, No. 661 (2001), pp. 299-306.

(3) Kaihara, T. and Fujii, S., A Study on Partnering Strategy in Virtual Enterprise (Game Theoretic Approach), Transactions of the Japan Society of Mechanical Engineers, Series C, Vol. 69, No.680 (2003), pp. 317-324.

(4) Nishi, T., Distributed Optimization Technique for Supply Chain Management, Journal of the Japan Society for Artificial Intelligence, Vol. 19, No.5 (2004), pp. 571-578.

(5) Emerson, D. and Piramuthu, S., Agent-based Framework for Dynamic Supply Chain Configuration, Proc. of the 37th Hawaii International Conference on System Science (2004), 70168a, CD-ROM.

(6) Piramuthu, S., Knowledge-based Framework for Automated Dynamic Supply Chain Configuration, European Journal of Operational Research, 165 (2005), pp. 219-230.

(7) Tanimizu, Y. et al., Multi-Agent Based Dynamic Supply Chain Configuration Considering Production Schedules, Proc. of 2006 International Symposium on Flexible Automation (2006), pp. 572-578.

(8) Tanimizu, Y. et al., Credibility of Supplier in Dynamic Supply Chain, Proc. of the 40th CIRP International Seminar on Manufacturing Systems CD-ROM, SC5 (2007), pp. 1-6.

(9) Holland, J. H., Adaptation in Natural and Artificial Systems, University of Michigan Press (1975).

(10) Tanimizu, Y., et al., Genetic Algorithm Based Reactive Scheduling, 1st Report, Modification of Production Schedule for Delays of Manufacturing Processes, Transactions of the Japan Society of Mechanical Engineers, Series C, Vol. 69, No. 685 (2003), pp. 234-239.

(11) Tanimizu, Y., et al., Evolutional Reactive Scheduling for Agile Manufacturing Systems, International Journal of Production Research, 44, 18-19 (2006), pp. 3727-3742.

(12) Lawrence, S., Resource Constrained Project Scheduling: An Experimental Investigation of Heuristic Scheduling Techniques (Supplement) Carnegie Mellon University, Pennsylvania (1984). 